Brit. J. Ophthal., 35, 284.

\title{
INTRACAPSULAR EXTRACTION BY KIRBY'S TECHNIQUE* RESULTS IN SIXTY CASES
}

BY

\author{
JAMES R. HUDSON
}

London

THE principles underlying Kirby's method of intracapsular extraction (Kirby, 1949) seemed to be mechanically sound, and it was decided to take advantage of the opportunity which I was privileged to have as a house surgeon at Moorfields Hospital, to make use of this technique. With the exception of a small number of cases operated on recently at the West Middlesex Hospital, the results given refer to patients operated on at Moorfields between January and November, 1949. The cases are not consecutive, as the intracapsular operation was not undertaken where it was considered that the extracapsular procedure would give an equally good visual result, and in a few cases alternative methods of intracapsular extraction were used. The stages of the operation will be briefly described and mention will be made of the use of a modification of Greeves' conjunctival stitch (Greeves, 1933) as an aid to security of the wound.

\section{METHOD}

The preliminary measures of culture, pre-operative use of penicillin and proflavine drops, sedation, and general preparation of the patient are those normally employed at Moorfields Hospital. Mydriasis is achieved by the pre-operative instillation of 2 per cent. homatropine, and if the pupil is not fully dilated by the time the patient reaches the operating theatre, adrenaline ( 3 minims $1 / 1000$ solution) is injected subconjunctivally, in the 6 o'clock position.

ANAESTHESIA.-Local anaesthesia is effected by:

(1) the instillation of 4 per cent. cocaine drops;

(2) retrobulbar injection of $1.5 \mathrm{ml}$. 4 per cent. novocaine.

The latter injection is given through the upper eyelid, the needle being introduced along the medial border of the superior rectus and then directed downwards and backwards into the muscle cone. The advantages of this technique are:

(1) the low incidence of retrobulbar haemorrhage-this complication did not occur in any of the cases described;

(2) the production of akinesia of the superior rectus by direct infiltration.

Akinesia of the muscles of eyelid closure is induced by the direct injection of those branches of the seventh nerve supplying the lid muscles near the condyle of the mandible (O'Brien, 1929).

* Received for publication February 13, 1951. 
TECHNIQUE

Lid Retraction.-Three black silk sutures are used, two in the upper lid and one in the lower lid (Fig. 1).

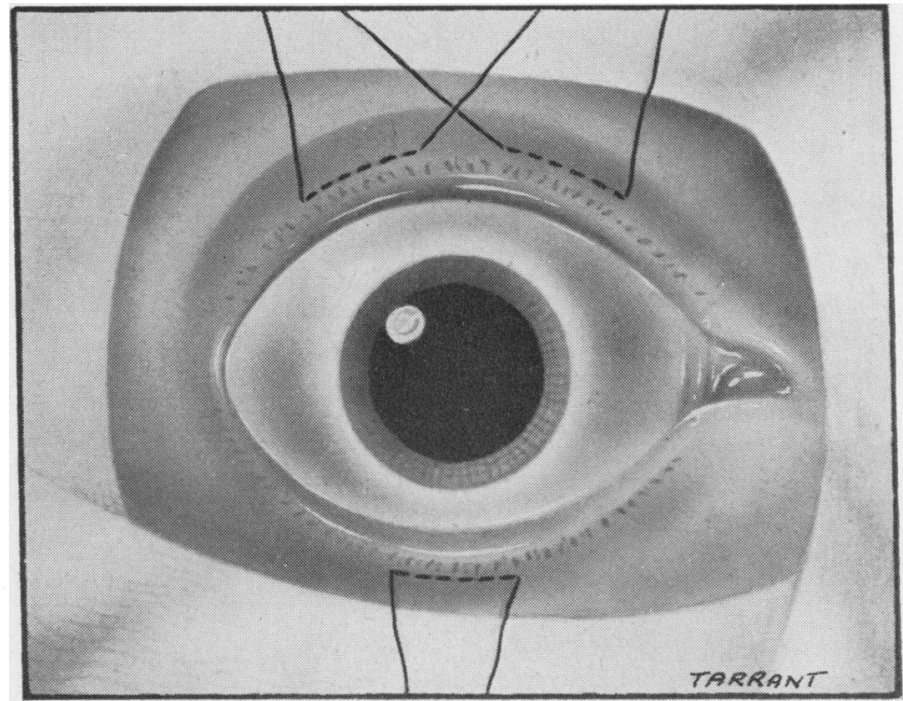

FIG. 1.-Lid-retraction sutures in position.

Fixation of Eyeball.- This is secured by a white silk suture through the superior rectus muscle, and fixation by forceps of the tendon of the internal rectus (Fig. 2).

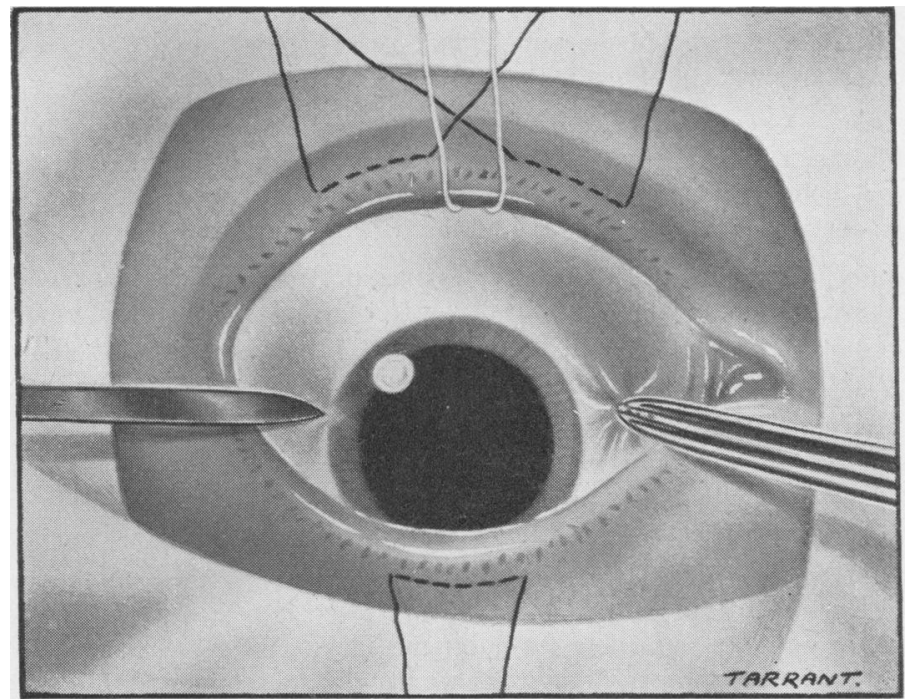

FIG. 2.-Fixation, using superior rectus stitch and fixation of medial rectus insertion by forceps. 


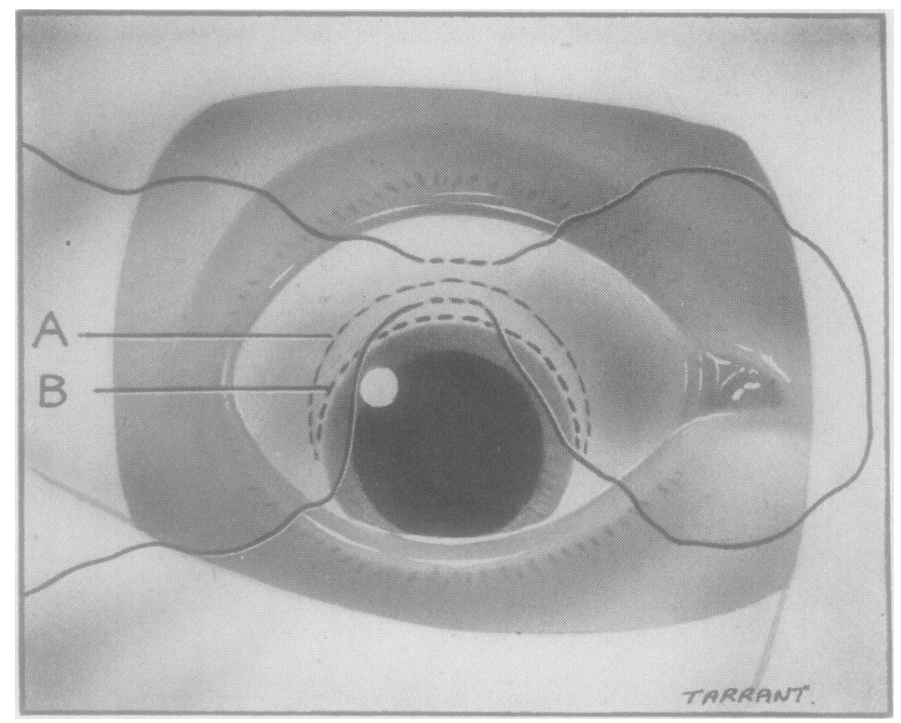

FIG. 3.--Modification of Greeves' conjunctival stitch. Upper limb of stitch passes superficially through sclera.

A. Line of conjunctival flap. B. Line of sclero-corneal incision.

Section. A large corneal section is made with a Graefe knife, beginning across or just above the horizontal meridian. In three cases a corneo-scleral stitch was used (Stallard, 1938), in 33 cases a modification of Greeves' conjunctival stitch was used (Greeves, 1933), the upper limb of the stitch being scleral rather than conjunctival in its insertion (Fig. 3), and in the remaining 24 cases a conjunctival flap was cut on completing the section, but no stitch was introduced.

Iridectomy. - A peripheral or complete iridectomy is then performed, depending upon the circumstances encountered at the time of operation, and the anterior chamber is washed out if bleeding from the section has obscured the view of the lens. At this stage the superior rectus suture is released, but is left in position.

Application of Forceps.--Castroviejo's cross-action forceps are used, and these are applied above, tangentially, just in front of the true equator of the lens (Verhoeff, 1927) (Fig. 4).

Removal of Lens. - The zonule is ruptured by the indirect method as described in detail by Kirby (1949), but using an Arruga's lens expressor. The lens is held in the forceps, gentle traction being exerted until the rupture of the zonule has been completed, when the upper margin of the lens will appear in the section. The expressor is then used to follow up the lens as its removal through the section is completed.

Completion of Operation.-The iris carefully reposed and the section closed, the details of technique depending upon whether a stitch has been used. The superior rectus suture and the retraction stitch on the lower eyelid are removed. Eserine ( 1 per cent. aqueous) and penicillin drops are instilled, and the upper lid is secured in the closed position by means of the retraction sutures placed at the beginning of the operation. 


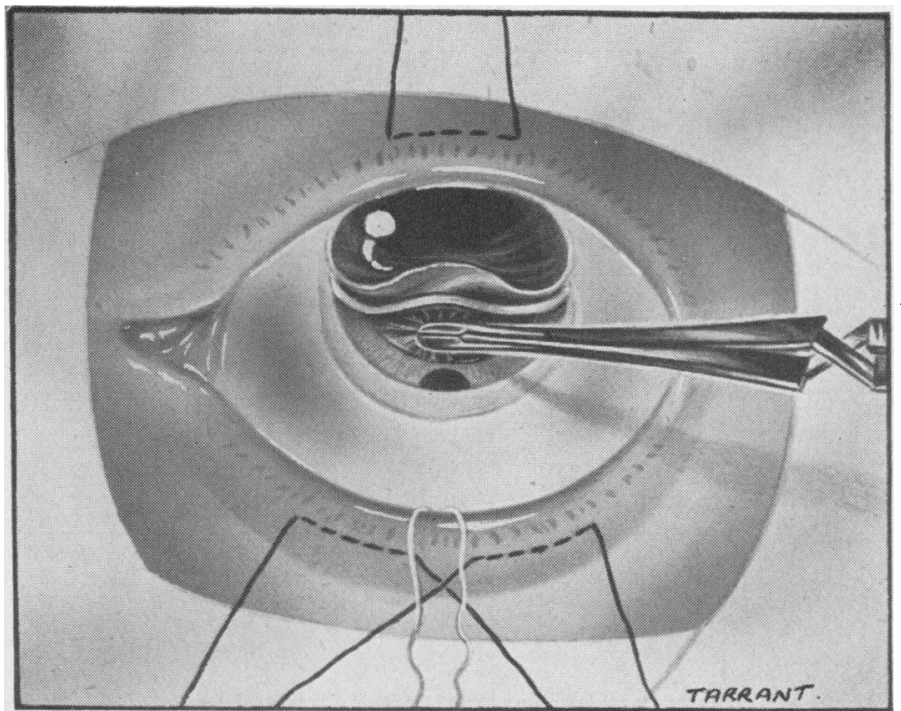

Fig. 4.-The capsule is grasped tangentially near the equator of the lens in the 12 o'clock position (seen from above).

Post-operative Care.-The post-operative treatment follows the lines normally adopted after intracapsular extraction. The patient is allowed to sit out of bed on the third post-operative day, and occasionally on the second day, and this greatly increases his]comfort without detriment to the healing of the eye. It also minimizes the risk of the major general complication of pulmonary embolism in older patients.

\section{RESULTS}

\section{Pre-operative Complications}

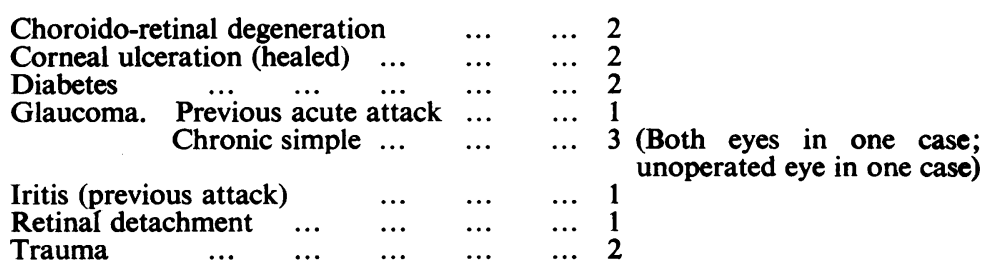

Operative Complications

$\begin{array}{lrrrrrr}\text { Vitreous loss } & \ldots & \ldots & \ldots & \ldots & \ldots & 0 \\ \text { Rupture of capsule } & \ldots & \ldots & \ldots & \ldots & 6\end{array}$

Arruga's forceps used in two cases.

Castroviejo's forceps used in four cases.

Capsule removed separately in four cases.

Capsular remnants seen post-operatively in two cases. 
Post-operative Complications

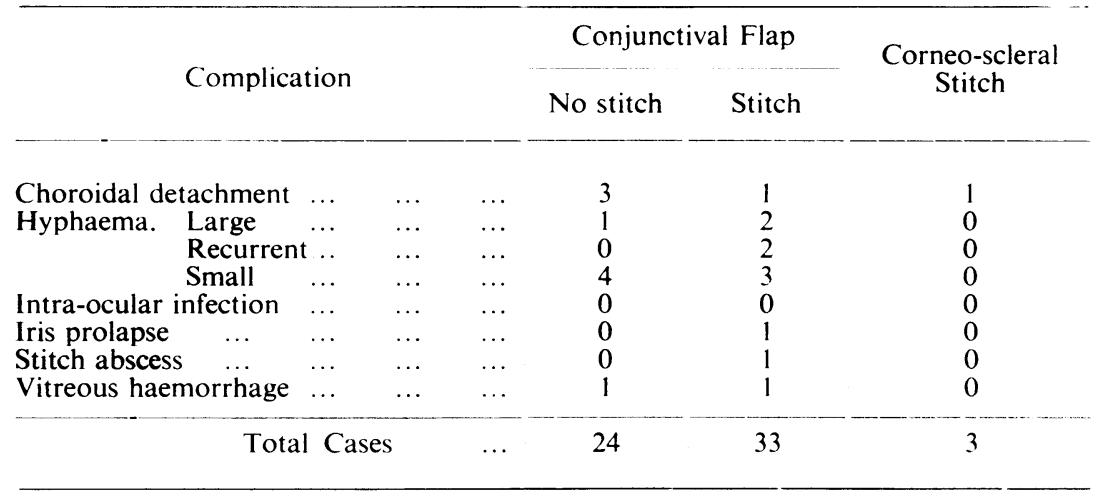

Post-operative Visual Acuity,

\begin{tabular}{|c|c|c|c|c|c|c|c|c|c|}
\hline Grade & $\ldots$ & $6 / 5$ & 66 & $6 / 9$ & $6 / 12$ & $6 / 18$ & 624 & $<624>6 / 60$ & $6 / 60$ or less \\
\hline No. of & & 2 & 4 & 22 & 14 & 8 & 3 & 2 & 5 \\
\hline
\end{tabular}

Causes of Reduced Visual Acuity (less than 6/12)

\begin{tabular}{llc}
\hline \multirow{2}{*}{ Opacities of Media } & Cornea & 2 \\
\cline { 2 - 3 } & Vitreous & 2 \\
\hline Retinopathy & Arteriosclerotic & $1^{*}$ \\
\cline { 2 - 3 } & Diabetic & 0 \\
\cline { 2 - 3 } & Senile macular degeneration & 2 \\
\cline { 2 - 3 } & Myopic fundus changes & 2 \\
\hline Retinal Detachment & Secondary & 1 \\
\hline Glaucoma & Chronic simple & 1 \\
\hline Unknown & & 2 \\
\hline Total & & 6 \\
\hline
\end{tabular}

* One case with central corneal nebula and arteriosclerotic fundus changes 


\section{COMMENTARY}

Kirby classified the various manoeuvres for rupture of the zonule as:

(1) Indirect Rupture by:

(a) pressure from without;

(b) traction on the lens or its capsule and transmitted to the zonule;

(c) rotation of the lens in its capsule;

(d) various combinations of these manoeuvres.

(2) Direct Rupture, used alone or in combination with one of the above methods.

In the present series, direct rupture of the zonule was not attempted. This technique might have prevented the rupture of the lens capsule in the six cases in which this occurred, but on the other hand, if the lens capsule is thin, the indirect method can still be successfully used, providing that the greater force is produced by pressure from without, the capsule forceps being used as a guide for the emerging lens rather than to assist in the rupture of the zonule.

In all but two cases (in which Arruga's intracapsular forceps were employed) Castroviejo's cross-action forceps were used (Castroviejo, 1939). These forceps have the following advantages:

(1) having gripped the capsule, the surgeon need exert no muscular effort to maintain the hold, and the small muscles of the hand are free for delicate manipulation;

(2) an equal bite of capsule can always be taken by opening the forceps as far as the stop;

(3) a uniform pressure is exerted by the blades of the forceps, through the spring action of the instrument.

The use of Verhoeff's technique (Verhoeff, 1927) of holding the lens above has two important advantages:

(1) a good view is given of the progress of the extraction;

(2) as the lens is slid rather than tumbled out, there is little disturbance of the vitreous face. This is well brought out by Harrington (1948), and the absence of vitreous loss as an operative complication in the present series of cases gives practical support to his view.

The post-operative complications can only be compared between those cases in which a conjunctival flap alone was used, and those in which a conjunctivo-scleral stitch was introduced, as a corneo-scleral suture was used on only three occasions. The incidence of hyphaema is much the same in both groups, and the one case of iris prolapse occurred in a patient in whom a stitch was used.* The stitch abscess which developed was in the sclera, and proved very resistant to treatment. There seems to be little correlation between the type of wound closure used and the incidence of choroidal detachment.

\footnotetext{
* This patient got out of bed and sat on the floor on the first post-operative day !
} 
The final visual results in the present series of cases emphasize the fact that, although 28 of the patients had a corrected acuity of $6 / 9$ or better, there is a significant number in whom the final vision was $6 / 12$ or less, in whom no obvious cause could be found. I have observed that, after intracapsular extraction, the corrected visual acuity at the time of leaving hospital is generally lower than in cases of extracapsular extraction in whom only a fine posterior capsule remains. It would not be unreasonable to assume that the intracapsular procedure causes considerably more temporary disorganization of the globe than does the more conservative type of operation. irrespective of the particular technique adopted.

\section{SUMMARY}

A series of sixty cases is reviewed, in which intracapsular extraction was performed by Kirby's technique. The operative results, complications, and corrected visual acuities are tabulated.

I should like to thank the members of the Consultant Staff at Moorfields for their encouragement, and for permission to make use of the hospital records of the cases. A few patients were operated upon at the West Middlesex Hospital, and my thanks are due to Mr. R. L. Galloway, the Medical Director, for permission to use the case records.

\section{REFERENCES}

Castroviejo, R. (1939). Trans. Amer. Acad. Ophthal. Otolaryng., 44, 394. GreEveS, R. A. (1933). Trans. ophthal. Soc. U.K., 53, 86.

Harrington, D. O. (1948). Trans. Amer. ophthal. Soc., 46, 294.

KIRBY, D. B. (1949). British Journal of Ophthalmology, 33, 3.

O'Brien, C. S. (1929). Arch. Ophthal., Chicago, 1, 447.

Stallard, H. B. (1938). British Journal of Ophthalmology, 22, 269.

VerhoEfF, F. H. (1927). Trans. Amer. ophthal. Soc., 25, 54. 